\title{
Designing a Cross-Platform Mobile Learning System
}

\author{
Majid Abarghooei
}

\begin{abstract}
According to the recent advancement of smartphones and their various capabilities, the ubiquitous use of these gadgets is totally considerable in many aspects of our lives. One of the most important fields in which smartphones play an indispensable role in these days, is education. In this paper we are going to address the software architecture of an empirical cross platform mobile learning system which has been designed and implemented as value added service of Rightel Communication Service Company, under observation of MTIC department (Mobile Technology and Innovation Centre). The main purpose of this paper, as it was previously mentioned, is addressing software architecture of aforementioned mobile learning system and challenges which we encountered. We also present a novel architecture to meet the requirement of such a challenging system.
\end{abstract}

Index Terms-Design pattern, mobile learning, software architecture, software engineering for mobile.

\section{INTRODUCTION}

"Mobile learning - as we understand it - is not about delivering content to mobile devices but about the processes of coming to know and being able to operate successfully in, and across, new and ever changing contexts and learning spaces" [1]. Mobile learning has many definitions in the literature but they revolve around the mobility of the technology or the mobility of the learner [1]. Mobile devices have some characteristics which make them suitable for educational purposes: presence (psychological environment), location (physical environment) and communities (social environment) [1]. Hence, we try to design a mobile learning system benefiting from these characteristics and considering Frame model. "The Frame model describes a mode of learning in which learners may move from different physical and virtual locations and thereby participate and interact with other people, information, or systems - anywhere, anytime. [2]"

There are many universities and institutes which have online courses and provide their students with e-books and other electronic resources. The mobile learning system about which we are talking has been introduced as a useful tool for those kinds of students who prefer to deal with their assignments and courses using their handheld devices such as smartphones, tablets and etc. The main purpose of this paper is presenting the architecture on which this cross platform mobile learning system has been designed and implemented;

Manuscript received April 30, 2014; revised August 5, 2014. This work was subsidized by the MTIC Department of Rightel Communication Service Company.

Majid Abarghooei is with Islamic Azad University South Tehran Branch, Software Engineering Department, Tehran, Iran (e-mail: m.abarghooei@aol.com). hence, we are not talking about the implementation of this system in detail and only some of the applied technologies will be mentioned. The organization of the paper is as follows: first of all in this paper, we are going to have an introduction to this system and all of the functionalities each of which affects the architecture of this system. After that we present the outline of the system, its architecture, and challenges which has been overcome in this system. From now on we are going to abbreviate this system as CPMLS (Cross Platform Mobile Learning System).

\section{CPMLS DEFINITION AND FUNCTIONALITIES}

In this section we are going to introduce the functionalities of CPMLS. Hence, first of all we present our expectations of this system. This system is supposed to facilitate the education process by providing the students with tools which bring them following features: being in contact with their classmates and professors, accessing educational resources, following announcements and news, watching video lectures on the go, reviewing new assignments, and even doing quiz tests; On the other hand, professors and other educational consultants involving in the education process have some requirements and expectations which should be met. They should be able to do the following tasks: adding new assignments, making quiz-tests, inserting grades, and adding resources. It is clear that the institute has some requirements such as managing students, professors and courses information. According to these requirements, the system should involve two major parts:

1) A multi-platform web-based mobile application.

2) Administration panels with which we are able to provide the application with proper information.

Each of the aforementioned parts has their requirements. In what follows, both of them are discussed and outline of their functionalities shall be delineated.

\section{CPMLS MOBILE APP}

According to the main purpose of designing CPMLS, it should be applicable to smartphones and other handheld devices. Hence, we should design a mobile application which can be deployed in popular operating systems which are widely used in handheld devices. This mobile application should meet following requirements:

1) Announcements: this section involves announcements and latest news which are provided for the students by the professor or institute.

2) Video Lectures: Through this section, students can find video lectures of all of the classes related to their courses and watch them.

3) Quiz-Tests: All of the professors can make quiz-tests 
and students can choose the related test and try to answer the questions. For each attempt which they make, a separate score will be saved.

4) Grades: In this section all of the students become informed of the grades which they have achieved in each course by detail.

5) Message Box: This app provides the students with the ability to send private messages to their professors and class-mates.

6) Chat: In this section all of the students using the app can chat with their friends and class-mates and discuss about their courses.

7) Resources: In this section students can access the resources which have been provided for them such as e-books and presentation files (PowerPoint) and download them to their smartphones or tablets.

8) Assignments: In this section of the app, students can access and download the assignments which their professors have assigned to them.

9) Consultancy: This part of the app contains a list of consultants which is provided by the institute. This list contains names and contacts of consultants. Students are able to choose one of the consultants according to their field of study and call them, using the app.

\section{CPMLS ADMINISTRATION PANEL}

As it was stated formerly, administration panels are used to provide the mobile application with proper information. All of the users working with the system should be provided with an account and have a role. We consider five roles in this system: STUDENT, PROFESSOR, CONSULTANT, ADMIN, and MANAGER. The first role belongs to students and is applied mobile application. Other four roles are used in administration panels each of whom has a specific authority. We divide the administration panel into three parts according to the requirements mentioned before and existing roles in the system.

\section{A. Professors}

The first part of the administration panel, which we want to address, belongs to professors' tasks. When a professor logs in to his account via the username which has been provided for him, three options are accessible for him according to his role in the system:

- Making Quiz-Tests: In this part he can choose the related course and then insert questions and answers. Note that he can only add single or multiple choice questions.

- Inserting Grades: In this section the professor first adds the title of the grades (e.g. mid-term exam, final exam, projects, papers, etc.) and inserts student grades.

- Adding News and Announcements: In this section the professor is able to add announcements and apprise students of news and important information (e.g. seminars, schedule of review classes, deadline of papers or assignments, etc.). These announcements are shown in the mobile app with the name of the professor and the date and time in which the news has been submitted.

- Posting Assignments: In this part the professor is able to post assignments and homework which are to be done by students. In this way he can upload assignments in different formats such as PDF files. All of the assignments are shown in the mobile application in related category.

- Posting video lectures: This part gives the professor the ability of uploading video lectures related to each class. All of the uploaded videos are show in the mobile application in the related class and students can choose and watch each of the videos.

- Adding Resources: In this part the professor can add resources (e-books, papers, etc.) which students have to study in the related course or use them for further studies.

\section{B. Institute's Administrators}

Each institute can have one or more administrators which are responsible for tasks some of which were mentioned in the requirements of the system and here we address them in detail.

- Adding News and Announcements: This part is exactly identical to the announcement part existing in the professors' account. Note that, in the mobile application, announcements which are posted by administrators are visually different from those are submitted by professors.

- Adding Programs: All of the Programs which exist in the institute and their information should be added in this section.

- Adding Courses: Each program has at least one course. In this section the administrator can add courses and assign each of them to its related program.

- Registering Students: This section gives the administrator the ability of registering new students. The administrator can either register students one by one or use batch register. In the latter way they can upload a file containing a list of new students to expedite the process of registering. The administrator is also able to assign all of the courses each student has taken.

- Adding Professors' Information: The administrator should use this part in order to add professors' information. This section have batch upload feature like the previous part. Note that the administrator should assign all of the courses taught by the professor to the same professor.

\section{Managers}

Institute's manager(s) can check the system status or management reports using their account. Functionalities of this system highly depends on the institute's policy because types of these reports varies from one institute to another according to their main concerns.

\section{ARCHITECTURE OF CMPLS}

In last section we tried to have an introduction to CPMLS and its outline and functionalities. Now we are going to address the architecture of the CPMLS. The first and the most important part of the system, as it was contended before, is its mobile application which should be cross-platform.

There are three solutions to have a cross platform mobile application:
A. Designing a Specific App for Each of the Operating Systems on Which We Have Intend to Deploy This
Application 
For example if we decide to deploy this app on iOS, Android, and Windows phone, we should implement three different native applications each of which should be implemented with a specific programming language (Objective C, Java, C\#, etc.) and an IDE which makes this a time consuming process. In this solution it is hard to design an integrated user interface for all of these operating systems because each of which provides different tools which is not identical to one another. There is another disadvantage to this solution. Whenever we decide to update the application, we should change native codes for all of the three operating systems, which were mentioned above, and export a new app. This means that all of the students should download the new version of the application according to the OS of their smartphones or tablets.

\section{B. Using Frameworks Which Make Us Able to Develop a} Cross-Platform Mobile Application and Access Device Native Functions without Dealing with Problems Mentioned in the Previous Solution

These kinds of SDKs (such as Apache Cordova, PhoneGap, and Titanium) require the use of JavaScript, HTM, and CSS from programming and export a native app for all of the widely used mobile operating systems [3], [4]. Although using web technologies such as JavaScript, HTML and CSS gives the flexibility in designing user interface, developing this app using these kinds of technologies is inappropriate for such a scale because not only have they some limitations, but also they have various kinds of methods which are so time-consuming to learn. It still has the problem of updating the application and every time the application needs a simple modification all of the users need to download the app.

\section{Using Hybrid Mobile Application}

According to the functionalities of the mobile application of CPMLS, we do not need the system services of the device which are accessible through native codes or few of them are available in SDKs discussed in previous solution. Hence, we design this application as a web application: we suppose that we are designing a mobile-optimized web application. This is where SDKs discussed in previous solutions are used. We use these frameworks to connect to the web application which has been developed. However, the user does not realize that he is using a web application because we have developed a light cross-platform mobile application which provides us with a web-view frame (which acts as a built-in browser) that connects to the web application. In this way all of the modification can be applied to the system without requiring users to download a new version of the app.

The cross-platform SDKs are also used in this solution to check the internet connection of the handheld device and other control functions which do not contain sophisticated programming and algorithms. Another advantage of this solution is that the size of this app is considerably smaller than those which have been developed by native codes. In this way we have both the flexibility in designing user interface and ease of implementation via web technologies and design patterns which make the development process faster and more comfortable. This is the solution we apply in this system to develop a cross-platform mobile application.
Fig. 1 depicts the outline of the hybrid mobile application of CPMLS.

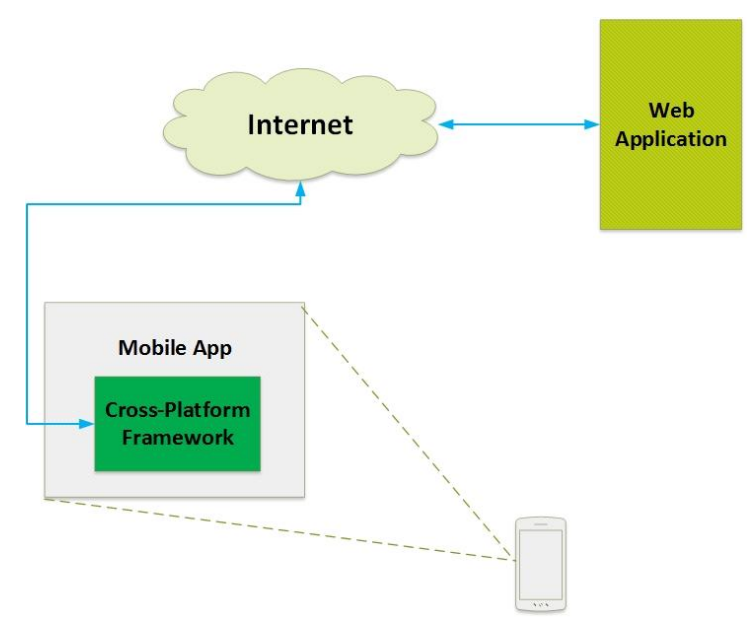

Fig. 1. Outline of the hybrid mobile application of CPMLS.

Both of the web applications in this system (administration panels and mobile web application) have 3-layer hybrid architecture using MVC design pattern in presentation layer (each layer can potentially run on a different machine).

In order to implement this architecture we divide each web application into two major modules completely independent of each other. We use Maven technology to build each module. These modules are as follows:

1) Core Module: This module covers our service layer and data access layer [5].

2) Web Module: This module covers our presentation layer which MVC pattern is applied to [5].

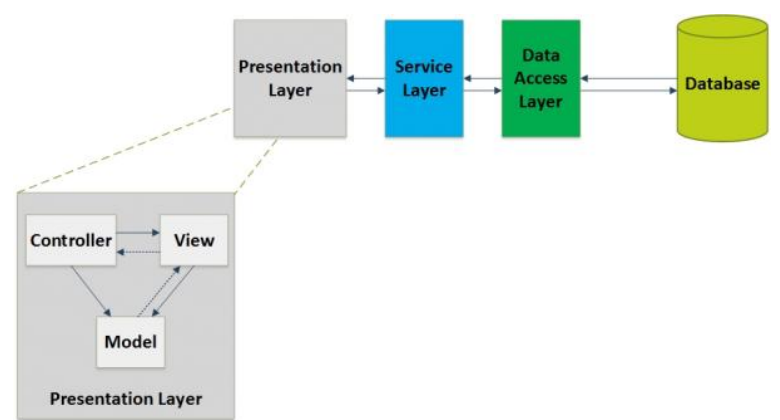

Fig. 2. The outline of the architecture of CPMLS's web applications.

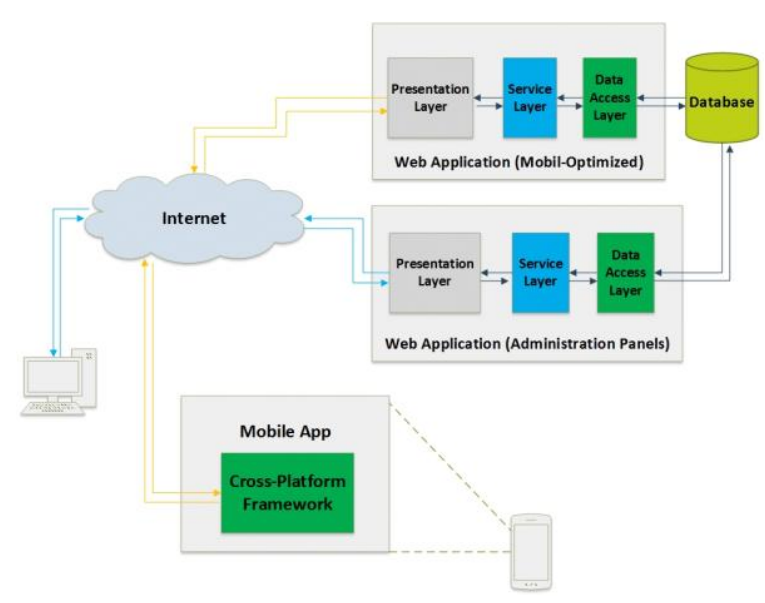

Fig. 3. Architecture of the CPMLS

Note that in order to reduce the coupling of the each module we have applied "Dependency Injection"; thereby, we use Spring framework for this purpose and also use its 
transaction management. For authentication and authorization purposes we benefit from Spring Security [6], [7]. We use Spring's Web MVC framework to apply MVC pattern in the application layer [8]. Fig. 2 and Fig. 3 present the outline of the architecture of CPMLS's web applications and its general architecture respectively.

Now it is time to stick the all of the previous parts together.

However there is another important module which has not yet been presented. What about the upload and download process? This is what we discuss in the next section.

\section{ChallengeS}

Suppose that there are thousands of students using the mobile app. They watch their classes online, download resources and etc. This considerably increases the workload of the web server. Hence, we should add a new server called "Content Server". We can use a web server which is more optimized for this purpose such as Nginx on the content server. In this way the workload of the web server would highly reduce and level of maintainability and reusability of both servers would increase. This is an important challenge in CPMLS because we should consider a mechanism to upload static files such as video lectures and resources to the content server.

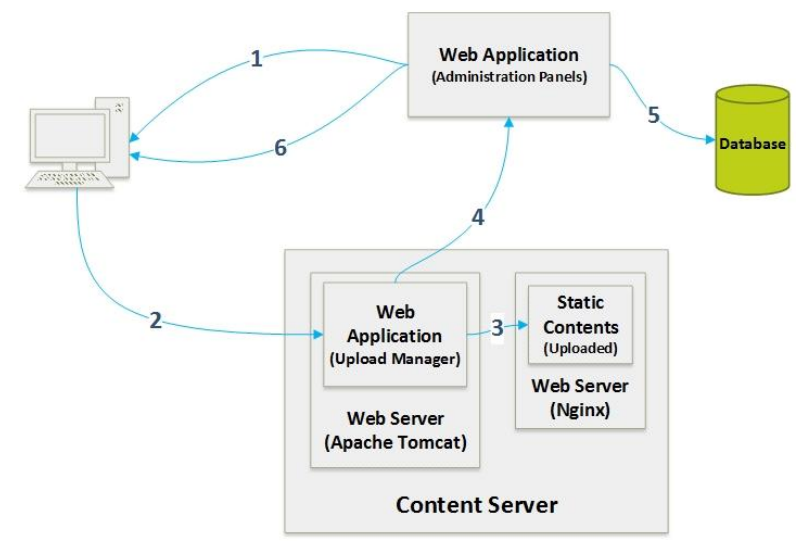

Fig. 4. Uploading sequence and content server.

In this way we develop a simple web application (using MVC in presentation layer) on content server called "Upload Manager" which is served by an identical web server with one we use for other web applications in this system. 1) Whenever a user (administrator, professor, etc.) intends to upload a file (video lecture, e-book, etc.), the administration panel sets the IP address of the content server. 2) Now the controller of the upload manager receives the request and 3) uploading process continues. 4) After that the upload manager redirects to the web application and 5) it saves the relational address of the uploaded file in the database. Note that the absolute address of the directory in which uploaded files are saved can be set in a XML file which makes the content server so flexible. What about the download process? When a user wants to download a file, Nginx gets the request on a specific port and URL pattern.

Now there is still another problem to solve. What if another person out of the system tries to upload files and makes the system crashed? To solve this problem we use RSA algorithm. We generate a pair of public and private keys for the upload manager. The web application which handles administration panels, signs the data of the file with public key and adds the signature to the view (e.g. a jsp file) which is rendered and sent to the client as HTML. When the user uploads the file, the signature is posted to the upload manager as well. The upload manager checks the signature with its private key. After that, allows the client to upload a file. In this way the aforementioned problem is solved. Fig. 4 depicts the uploading sequence.

\section{CONCLUSION}

According to the previous sections, we have a mobile learning system which consists of a cross-platform mobile application and a web application which handles administration panels. In this paper we tried to present a good-practice architecture which is a result of six-month effort in an empirical project. As it was stated in the introduction, we did not address the implementation of the system except introducing some technologies. Actually the solution presented above has been considered for this system; hence, applying these architectures to other systems with different requirements needs research and consideration.

\section{ACKNOWLEDGMENT}

F. M. Abarghooei thanks Dr. Khodayar Sarfaraz, former mobile technology and innovation consultant at MTIC departent of Rightel Communication Service Company, for his consideration and invaluable comments.

\section{REFERENCES}

[1] N. Pachler, B. Bachmair, J. Cook and G. Kress, Mobile Learning: Structures, Agency, Practices, 1st ed., New York, NY: Springer, 2010, ch. 1, pp. 3-10

[2] M. Ally, Mobile Learning: Transforming the Delivery of Education and Training, 1st ed., Edmonton, Canada: AU Press, 2009, ch. 2, pp. 25-42.

[3] PhoneGap Supported Features. (April 2014). [Online]. Available: http://phonegap.com/about/feature.

[4] Apache Cordova. (April 2014). [Online]. Available: http://cordova.apache.org.

[5] L. Bass, P. Clements, and R. Kazman, Software Architecture in Practice, 3rd ed., Boston, MA: Addison-Wesley Professional, 2012, ch. 13.

[6] R. Harrop and C. Ho, Pro Spring 3, 1st ed., New York, NY: Apress, 2012, pp. 53-495.

[7] C. Scarioni, Pro Spring Security, 1st ed., New York, NY: Apress, 2013, pp. 27-273.

[8] C. Yates, S. Ladd, M. Deinum, K. Serneels, and C. Vanfletere, Pro Spring MVC: With Web Flow, 1st ed., New York, NY: Apress, 2012, pp. 51-106.

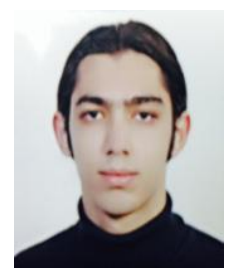

M. Abarghooei was born on November 30, 1987, in Tehran, Iran. He received his B.A. degree in software engineering at Islamic Azad University South Tehran Branch from 2006 to 2009.

In January 2011 he started working at Pars Rayan System Qeshm as a senior java developer. Next year he started working at Tourism Card Company as a software developer and IT consultant. He achieved Oracle Certification of Professional Java SE 6 Programmer (OCPJP 6) in January 2013. In June 2013 he started collaborating with Rightel Communication Service Company, MTIC department, as a Java developer and service contractor 